\title{
Changing trends in resistance pattern as an alarm by bacteria before it's too late to treat
}

\author{
Sumit Gupta ${ }^{2}$, Reshma Agarwal ${ }^{3}$, Suneel Bhooshan ${ }^{1}$, Manish Kumar Diwakar ${ }^{1}$, \\ Ankur Goyal $^{1}$, Arti Agrawal ${ }^{1}$ \\ 1(Microbiology Department S.N Medical College Agra Dr B.R. Ambedkar University Agra India) \\ 2(Microbiology Department JIPMER Pondicherry India) \\ 3(Microbiology Department Assam Medical College and Hospital. Dibrugarh, Assam India.'
}

\begin{abstract}
Background- Urinary Tract Infection is one of the most common site of bacterial infection particularly in female accounting for $2 o-3 o \%$ of women. UTI in men are less common and primary occur after 50 years of age. It is important to know the causative organisms in the hospital and community for optimum management of UTI.

Objectives-To investigate the prevalence and antimicrobial susceptibility of bacterial uropathogens from the patients attending S.N Medical College, Agra.

Methods and Material-The retrospective study involved 1022 UTI patient. Out of these 60\% were females and $40 \%$ males. Clean catch mid-stream urine from symptomatic UTI cases were analyzed in the microbiology laboratory, S.N Medical College, Agra. Bacterial isolate were identified using biochemical reaction. Antimicrobial susceptibility testing was performed by the Kirby-Bauer disc diffusion method as described in CLSI guidelines 2010.

Results - The overall prevalence of UTI was found to be $63.60 \%$ (650/1022). Klebsiella pneumonia (38.46\%) and Escherichia coli (42.76\%) were most common organism isolates.

Conclusion - The most effective antibiotic for Escherichia coli and Klebsiella pneumonia were gentamycin, amikacin and cefoperzon- sulbactum. Regular monitoring is required to establish reliable information about susceptibility pattern of urinary pathogen for optimal empirical therapy of patient with UTI.
\end{abstract}

Key-words: Antimicrobial Susceptibility Pattern, Prevalence, Urinary Tract Infection.

\section{Introduction}

Urinary Tract Infection (UTI) is one of the most important causes of long term morbidity and mortality with an estimated 150 million UTIs annually (Gobernado et al 2007). It is associated with renal damage reported in about $5 \%$ of affected children and is common both in the community and hospitalized patients ${ }^{[4]}$. Although UTIs are encountered in the both sexes and all ages but it is more common in females than the males. Uropathogenic Escherichia coli (UPEC) is the most common etiologic agent, responsible for 80 to $85 \%$ of community- acquired UTIs with other significant uropathogens; including Staphylococcus saprophyticus, Klebsiella pneumoniae, and Proteus mirabilis ${ }^{[9.10]}$. In present scenario, the essence of antimicrobial drug resistance of major uropathogens has posed a global threat. Updated knowledge of the prevailing etiological agent of UTI and their antimicrobial resistance patterns in specific geographical location may aid clinician in choosing the appropriate antimicrobial empirical treatment and development of appropriate drug policies. Therefore the present study was undertaken to find out the prevalence of UTI and to determine the antimicrobial susceptibility patterns of commonly used antibiotics among patient.

\section{Material \& Method}

2.1 Sample collection and analysis-This study was conducted in the Department of Microbiology S.N Medical College, Agra located in the Northern region of India from $1^{\text {st }}$ May 2012 to $1^{\text {st }}$ Feb 2013 on in patients and patients attending outpatient clinics. A total of 1022 patient with sign and symptoms of UTI were included for this study. All study subjects were advised to collect the freshly voided mid-stream urine sample in wide mouthed leak proof sterile container to hold about $50 \mathrm{ml}$ specimens. Samples from catheterization and suprapubic aspirate were also included in the study. The specimens were transported immediately within one hour to laboratory for further processing and to ensure that the pathogenic organisms present in the urine were isolated and also to avoid overgrowth of the pathogenic organism ${ }^{[2]}$.

2.2 Exclusion criteria - Exclusion criteria for patients were antibiotic usage within week and large fluid intake (less than one hour) before clinic attendance. Only a single positive culture per patient was included in the analysis. 
2.3 Direct microscopy: Well mixed, un-centrifuged $10 \mu$ l of urine were placed onto a glass slide and allow it to air dry without spreading and gram stain was performed. Each bacteria/oil immersion field indicate the count of $10^{5} / \mathrm{ml}$ of urine and $>5$ pus cell/HPF were consider significant to indicate infection. ${ }^{[2]}$

2.4 Urine culture: Semi quantitative urine culture technique $\{$ using a calibrated loop $(0.01 \mathrm{ml})\}$ was used to inoculate blood agar and Cystein Lactose Electrolyte Deficient medium (CLED). The plates were incubated for 24 hour at $37^{\circ} \mathrm{C}$. The bacterial count was expressed as colony forming units (CFU) per milliliter (ml). Following the recommendations of Kass in distinguish in the infection from contamination, significant bacteriuria was defined as culture of a single bacterial species from the urine sample at a concentration of $>10^{5} \mathrm{CFU} / \mathrm{ml}{ }^{[11]}$. Further a single organism was identified by standard biochemical test ${ }^{[1]}$.

2.5 Antibiotic sensitivity testing: Antibiotic sensitivity testing was performed using the Kirby Bauer disc diffusion method as described in the CLSI guidelines 2010. The in vitro susceptibility to isolated urinary tract pathogen were tested against amoxicillin, co-trimoxazole, cefixime, oflaxacin, tetracycline, ciprofloxacin, norfloxacin, Cefotaxime, cefadroxil, gentamycin, amikacin, cefoperazone-sulbacta , imipenem, nitrofurantoin. Interpretation of result was done by measuring the zone of inhibition around the antibiotic discs in millimeters. Escherichia coli ATCC 25922, Staphylococcus aureus ATCC 29213, Pseudomonas aeruginosa ATCC 27853 and Enterococcus faecalis ATCC 29212 were used as quality control strains for antimicrobial susceptibility. Statistical test: Microsoft office excel 2007 was used to analyze the data.

\section{Result-}

Of the 1022 urine specimens processed $650(63.60 \%)$ showed significant growth of pathogens. The patients were between new born and 90 years of age. Remaining 372 samples had either contamination or a very low bacterial count / sterile.

\subsection{Distribution Pattern of Isolates between the Sexes:}

More cases of UTIs were recorded among females $390(60 \%)$ than the males $260(40 \%)$. The differences noted in the distribution of pathogens among the sexes were significant.

Table 1: Prevalence of urinary tract infection in relation to age and sex of patients.

\begin{tabular}{|l|l|l|l|l|l|l|}
\hline Age & $\begin{array}{l}\text { Male } \\
\text { Examined }\end{array}$ & $\begin{array}{l}\text { Male Positive } \\
(\%)\end{array}$ & $\begin{array}{l}\text { Female } \\
\text { Examined }\end{array}$ & $\begin{array}{l}\text { Female } \\
\text { Positive }(\%)\end{array}$ & $\begin{array}{l}\text { Total Number of } \\
\text { Cases }\end{array}$ & $\begin{array}{l}\text { Total Positivity } \\
\%\end{array}$ \\
\hline $0-19$ & 143 & $73(51 \%)$ & 186 & $115(62 \%)$ & 329 & $188(57.14 \%)$ \\
\hline $20-49$ & 168 & $88(52 \%)$ & 288 & $197(68 \%)$ & 456 & $285(62.50 \%)$ \\
\hline $50-90$ & 116 & $99(85 \%)$ & 121 & $78(64 \%)$ & 237 & $177(74.68 \%)$ \\
\hline Total & 427 & $260(61 \%)$ & 595 & $390(66 \%)$ & 1022 & $650(3.60 \%)$ \\
\hline
\end{tabular}

\subsection{Bacterial Agents of Urinary Tract Infection:}

Of the 650 significant isolates, gram-negative rods accounted for $94.31 \%$ while gram-positive cocci accounted for the remaining $05.69 \%$ of the total pathogens. The Gram Negative Rods isolated were Escherichia coli, Klebsiella pneumoniae, Pseudomonas aeruginosa, Proteus mirabilis and Serratia marcescens. Escherichia coli alone accounted for $42.76 \%$ followed by Klebsiella pneumoniae $38.46 \%$ of the urinary isolates, Serratia marcescens was $7.23 \%$, Pseudomonas aeruginosa $3.53 \%$, with respectively Proteus mirabilis $2.31 \%$. Among gram positive pathogens S. saprophyticus was the major pathogen accounted for $3.07 \%$ followed by Staphylococcus aureus $1.69 \%$ and Streptococcus pyogenes $0.92 \%$. The pattern of bacterial agents isolated is as shown in table 2.

Table 2 Pattern of etiological agents isolated from Patients $\left(\mathrm{N}^{+}=650\right)$

\begin{tabular}{|l|l|l|l|}
\hline Organism & No. of isolate \% in male & No. of isolate \% in Female & Total \% \\
\hline Escherichia coli & $124(44.60 \%)$ & $154(55.40 \%)$ & $278(42.76 \%)$ \\
\hline Klebsiella pneumoniae & $98(39.20 \%)$ & $152(60.80 \%)$ & $250(38.46 \%)$ \\
\hline Serratia marcescens & $11(23.40 \%)$ & $36(76.60 \%)$ & $47(7.23 \%)$ \\
\hline Pseudomonas aeruginosa & $14(60.86 \%)$ & $9(39.14 \%)$ & $23(3.53 \%)$ \\
\hline Proteus mirabilis & $3(20.00 \%)$ & $12(80.00 \%)$ & $15(2.31 \%)$ \\
\hline Streptococcus pyogenes & $2(33.33 \%)$ & $4(66.67 \%)$ & $6(0.92 \%)$ \\
\hline Staphylococcus aureus & $5(45.45 \%)$ & $6(54.55 \%)$ & $11(1.69 \%)$ \\
\hline Staphylococcus saprophyticus & $7(35.00 \%)$ & $13(65.00 \%)$ & $20(3.07 \%)$ \\
\hline Total Number N $\mathrm{N}^{+}$ & $264(40.61 \%)$ & $386(59.39 \%)$ & 650 \\
\hline
\end{tabular}




\subsection{Antibiotic Resistance pattern in Gram Negative Bacilli:}

The antimicrobial Resistance pattern of Gram Negative Bacilli against selected antimicrobial agents is summarized in Figure 1.

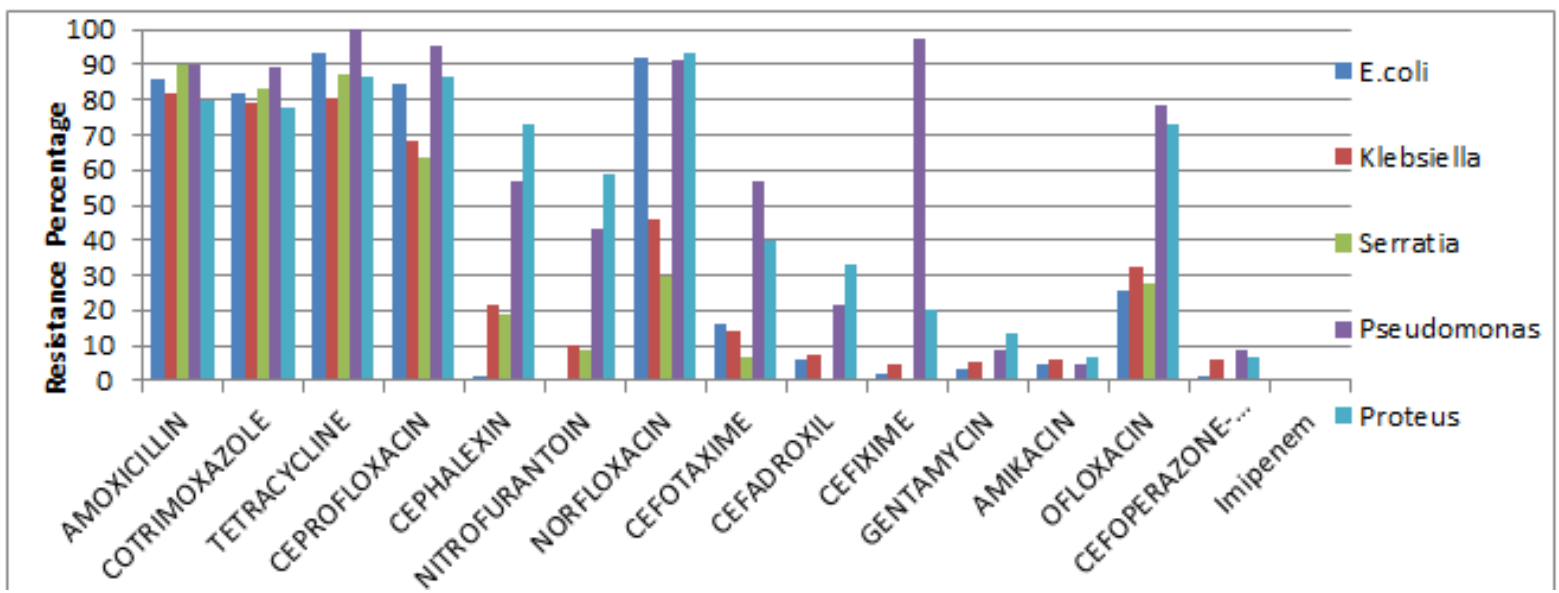

Fig 1 the antimicrobial Resistance pattern of Gram Negative Bacilli against selected antimicrobial agents.

\subsection{Susceptibility pattern of most common isolated Gram-negative bacteria}

E. coli and K. pneumoniae showed least resistance to the aminoglycosides - gentamicin $(0.27 \%$ and $6.05 \%)$, carbapenems $(0.31 \%$ and $0.46 \%)$ nitrofurantoin $(0.36 \%$ and $9.90 \%)$, cephalexin (1.08\% and $21.35 \%)$, cefoperazone-sulbactam (1.08\% and 5.69\%), cefixime (2.16\% and $4.98 \%$ ), cefdroxil (5.76\% and $7.47 \%$ ), cefotaxime (16.19\%and $14.23 \%)$, ofloxacin $(25.54 \%$ and $32.74 \%)$ respectively. Serratia was the third most common isolate in urinary tract pathogen which showed the similar resistance pattern like E.coli and $K$. pneumoniae in respective to amoxicillin , cotrimoxazole, tetracycline, ceprofloxacin, ofloxacin and it showed $100 \%$ susceptibility to cefixim, gentamycin, amikacin, and cefoperazone-sulbactam. While P. aeruginosa isolates showed the highest resistant towards routinely used antimicrobial and least resistance toward gentamycin $08.70 \%$, amikacin $4.35 \%$, cefoperazone-sulbactam $08.70 \%$. The highest susceptibility to aminoglycosides tested was also obvious with $P$. mirabilis isolates as shown in Fig1.

\subsection{Antibiotic susceptibility pattern of Gram Positive isolates:-}

The Gram -positive cocci were tested against - amoxicillin (AX), cotrimoxazole (BA), tetracycline (TE), ceprofloxacin (RC), cephalexin (CN), erythromycin (EE), azithromycin (AZ), rotxithromycin (RM), norfloxacin (NX), ofloxacin (NZ), ampicillin (AM), cloxacillin (CX), cefotaxime (CF), cefuroxime (CB), cefoperazne-sulbactam (CS), vancomycin (VA). S. pyogenes isolate were $100 \%$ resistance against Aamoxicillin, cotrimoxazole, tetracycline and susceptibility of amoxicillin-clavulanic acid $100 \%$, gentamicin $92.30 \%$, and ceftriaxone $84.60 \%$ respectively.

\section{Resistance \%}

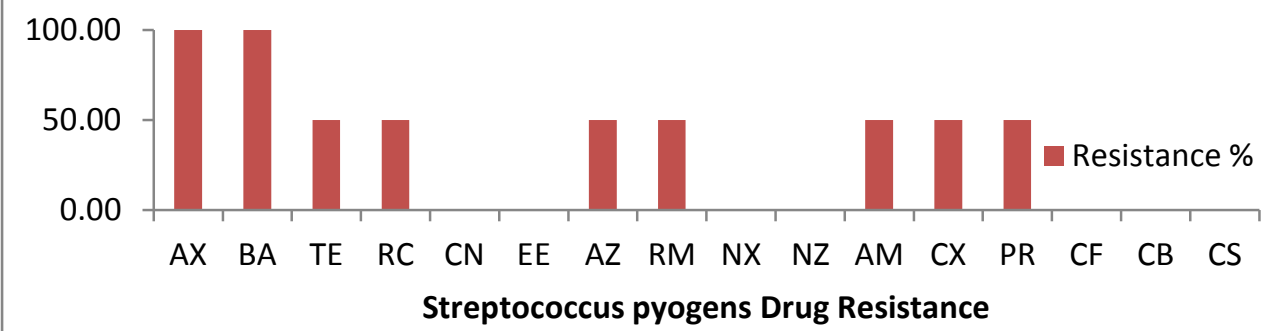

Fig 2 Streptococcus pyogenes drug resistance 
S. aureus showed highest resistant $54.54 \%$ to gatifloxacin, $45.45 \%$ to erythromycin and least resistance towards Gentamycin $27.27 \%$. S. saprophyticus isolate showed highest resistance towards erythromycin.

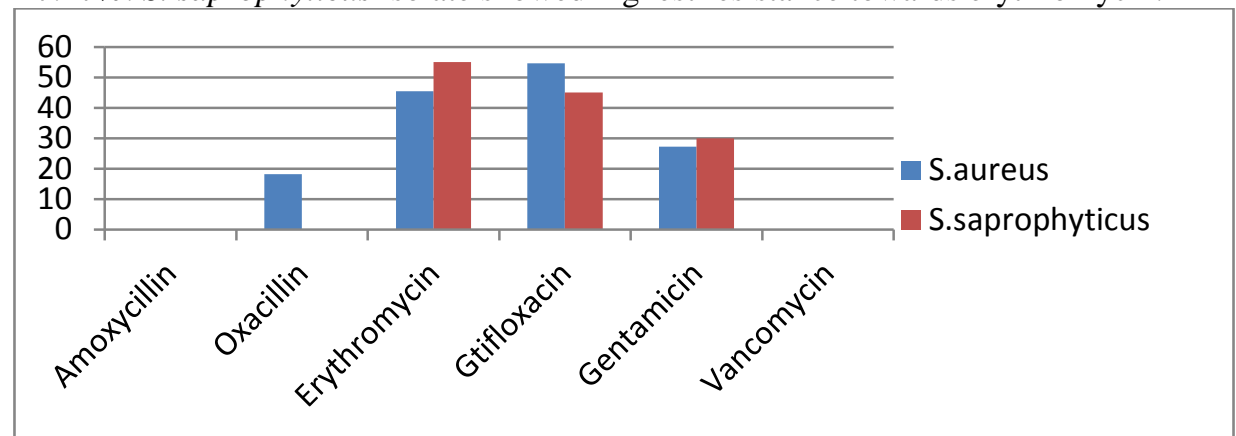

Figure 3 Drug resistance among other gram positives.

\section{Discussion-}

Although UTI ranks among the most common infection in developing countries, in the present study a total of $650(63.60 \%)$ out of 1022 patients had UTI. This indicates that urine culture and antimicrobial susceptibility is essential for a definitive diagnosis of UTI. Geographical location may be the reason for the difference in the percentage of population infected with UTI infection, the pattern of pathogenic organism and resistance pattern of different organism. According to other study prevalence rate of UTI was $24.94 \%$ Bharatpur ${ }^{[8] .}$ The prevalence of $39.69 \%$ was reported in okada, a rural community in Nigeria in $2011^{[7]}$.

In our study, UTI was more common in females. Male: female ratio was 1:1.46 as shown in table 2 . Other studies also showed male: female ratio of $1: 1.9$ to $1: 2^{\left[6,12 \text {, and }{ }^{15]}\right.}$ in different regions. Our result corresponds to the result obtained in similar study conducted in India, Kuwait, Sharjah and Schaeffer et al. [2001] too with $60 \%$ of infected population being women ${ }^{[13]}$. This is due to short urethra in females and its closeness to the anus. Sexual activity also appears to increase the chances of bacterial contamination of the female urethra.

The prevalence of antimicrobial resistance among micro-organism that causes UTI is increasing worldwide and is a major factor selecting antibiotics for treatment. In the present study, the most frequently isolate were E.coli, K. pneumoniae, S. marcescens, P. aeruginosa, P. mirabilis, S. aureus, S. saprophyticus and $S$. pyogenes. These were also the organism most commonly isolated India, Nepal, and Pakistan ${ }^{[9 a n d}{ }^{20]}$. In our study E.coli is the most common isolate in urine culture which was consistent with other study where $E$. coli is the most common isolate in urine culture ${ }^{[7,8]}$. The highest percentage of E.coli obtained in this study is a cause of concern due to emerging drug resistance. More females (154) were infected with E.coli with only 124 males. While in case of K. pneumoniae the number of infected females and males were 98 \& 152 respectively. Of the 47 isolates of Serratia, 11 were from males and 36 were from females which was significant (Table 2). Other organism isolated were $P$. aeruginosa (3.53\%) and P. mirabilis (2.31\%) as shown in Table 2 . These organisms also have been reported as agents of UTIs and their presence in the sample population was not unusual as showed by S Shrestha et al in children at Nepal Medical College Teaching Hospital, Nepal Medical College, Kathmandu, Nepal. In this study infection caused by $P$. aeruginosa was very common in males $60.86 \%$ than females which correlate with study conducted by B.V Ramana and Abhijit Chaudhary in 2011 in Andhra Pradesh.

Resistance rates vary from country to country ${ }^{[15]}$ this is probably due to the fact that the antibiotic has been widely used in treating UTIs over the past decade in the region. The antimicrobial sensitivity findings in our study was similar to a study conducted by Das et al. 2006, Tiwan, Senegal and India [ ${ }^{8,12 \text { and } 18]}$ that showed the greatest sensitivity to all the isolated organism against amoxacillin, cotrimoxazole, tetracycline, ciprofloxacin, ofloxacin and norfloxacin in comparison, low resistance rates were found against cephalexin, nitrofurantoin, cecfotaxime, cefadroxil, cefixime, gentamycin, amikacin and cefoperazone-sulbactamin Fig 1. The highest efficacy of gentamicin in the treatment of UTIs has also been reported by Al Sweih et al ${ }^{[14]}$ and the antimicrobial that showed the greatest resistance to the isolate organism were amoxicillin, cotrimoxazole, tetracycline and ciprofloxacin. The resistance pattern of $K$. pneumonia and E.coli was similar and higher for amoxicillin $(82.02 \% \& 85.56 \%)$, tetracycline $(80.78 \%$ \& 93.17), co-trimoxazole $(78.95 \% \& 81.76 \%)$, and ciprofloxacin $(84.57 \%$ \& 68.29\%). Our isolates showed higher resistance against ampicillin and co-trimoxazole than the isolates from USA (39.1\% and $18.6 \%$ respectively) ${ }^{[16]}$ and Europe $\left(29.8 \%\right.$ and $14.1 \%$ respectively) ${ }^{[17] .}$ Resistance rate for nitrofurantoin and carbapenems was very low (showed only $9.90 \% \& 0.36 \%$ resistance for nitrofurantoin and $0.46 \%$ and $0.31 \%$ for resistance for carbapenems) in $K$. pneumoniae and E.coli isolates respectively which was similar to major part of world ${ }^{[20] .}$ But $P$. mirabilis showed highest resistance towards nitrofurantoin 59\%. On the other hand rate of resistance against these antibiotics is comparable in countries like Senegal, Taiwan and India. ${ }^{[12,18]}$ Resistance pattern of S. marcescens isolates was similar to E.coli and $K$. 
pneumoniae isolates, where resistance percentage of ceprofloxacin was $64 \%$, cotrimoxazole $83 \%$, tetracycline $87 \%$ and for amoxacillin $90 \%$. These isolates were highly susceptible to other antimicrobials (Susceptibility varies from $70 \%$ to $100 \%)$. Fig 1

P. aeruginosa was resistant against Tetracycline 100\%, amoxicillin 90\%, cefixime 97\%, norfloxacin 91\%, ciprofloxacin $96 \%$ and co-trimoxazole $89 \%$ respectively. Resistance pattern in case of Proteus was also not unusual with highest resistance to, amoxicillin, tetracycline, norfloxacin, ciprofloxacin, and co-trimoxazole. imipenem had wide coverage for all isolates with almost $100 \%$ susceptibility among the all beta-lactam.

Percentage of Gram Positive isolates were very less in our study accounting for $05.69 \%$ of all the isolates Which was also similar to the studies conducted by Manjunath GN, Prakash R et al. from 2005 to 2010 and Zakieh Rostamzadeh Khameneh, Ali Taghizadeh $e t$ al. in 2006 Iran. However antibiotic resistance in Gram Positive cocci was observed from $0.00 \%$ to $100 \%$ as indicated on Fig 2 \& Fig 3. S. pyogenes isolates were 100\% resistance when they were tested against amoxacillin and cotrimoxazole. Isolates also showed $50 \%$ resistance towards erythromycin, azithromycin, tetracyclin, ciprofloxacin, rotxithromycin, cloxacillin, cefotaxime, cefuroxime and cefoperazone-sulbactam antibiotics showed good efficacy against gram positive isolates being $100 \%$ sensitive. Fig 2 Other gram positive isolates were S. aureus and S. saprophyticus which were tested against selective antibiotics and showed less resistance. S. aureus showed low level resistance towards gentamycin and erythromycin $27.27 \%$ and $45.45 \%$. Among 11 S. aureus isolates 2 were Methicillin Resistant. S. saprophyticus showed more resistance in comparison to $S$. aureus while tested against eythromycin and gentamycin. None of the Gram positive isolate showed resistance against vancomycin.

Most of isolated uropathogens showed multiple antibiotics resistance in this area. It may be due to large portion of the bacterial isolate being previously exposed to several antibiotics. The present study data gives idea about the common trend of increased antibiotics resistance of uropathogens in this region, which may be due to geographic variation or indiscriminate or sub lethal use of antibiotic.

The emergence and spread of resistance can be reduced through appropriate or careful use of antimicrobial drugs and increasing awareness among the population to the hazards of inappropriate antimicrobial use through public health education campaign.

\section{Conclusion-}

An overall prevalence $63.79 \%$ of UTI was observed in this study. This information will directly affect selection of empiric therapy for UTI. Regular monitoring is required to establish reliable information about susceptibility pattern of urinary pathogen for optimal empirical therapy of patient with UTI. We suggest that empirical antibiotic selection should be based on the knowledge of local prevalence of bacterial organism and antibiotic sensitivities rather than on universal guidelines.

\section{Reference}

[1]. Collee G, Duguid P, Fraser G, Marmian P. Mackey and MacCartney's practical medical microbiology 14th ed., Singapore: Churchill Livingstone Publishers. Longman; 2003.

[2]. Henry D. Isenberg, Clinical Microbiology Procedures Handbook,ASM Press Volume 1 second edition 2007.

[3]. Sood S ,Ravi Gupta, Antibiotic resistance pattern of community acquired Uropathogens at a tertiary care hospital in Jaipur, Rajasthan Indian J Community Med, 2012, Vol. 37,issue 1, 39 -44.

[4]. Kader AA,Kumar A, Dass SM. Antimicrobial resistance pattern of gram negative bacteria isolated from urine culture at a general hospital. Saudi J Kidney Dis Transpl2004:15(2):135-9.

[5]. S Iram, G Uma , EM Syed, A Jawed , Antibiotic susceptibility pattern of urinary pathogen in female outpatient. North American journal of medical science, april 2012, volume 4,issue (4) 163-169.

[6]. VU Muoneke, MU Ibekwe, RC Ibekwechildhood urinary tract infection in Abakaliki: etiological organism and antibiotic sensitivity pattern. Annals of medical health science research, 2012, vol2, issue (1),29-32.

[7]. HO Bankole, O Richard, O Mitsan,AA Joshua, urinary tract infection in a rural community of Nigeria. $N$ Am J Med Sci, 2011, Feb. vol 3. No. 75-77.

[8]. A Acharya, Gautam R, Subedee L. Uropathogen and their antimicrobial susceptibility pattern in bharatpur, Nepal.Nepal med coll .j. 2011 ,mar,13(1):30-3

[9]. Ronald, A. R., L. E. Nicolle, E. Stamm, J. Krieger, J. Warren, A. Schaeffer, K. G. Naber, T. M. Hooton, J. Johnson, S. Chambers, and V. Andriole. Urinary tract infection in adults: research priorities and strategies. Int J Antimicrob Agents. 2001 Apr; 17(4):3438.

[10]. Ronald A. The etiology of urinary tract infection: traditional and emerging pathogens. Dis. Mon.feb. 2003, 49 (2):71-82.

[11]. Girou E, Rioux C, Brun-Buisson C, Lobel B: The postoperative bacteriuria score: a new way to predict nosocomial infection after prostate surgery. Infection Committee of the French Association of Urology. Infect Control HospEpidemiol2006, Aug 27(8):84754.

[12]. Akram M, Shahid M, Khan AU. Etiology and antibiotic resistance patterns of community-acquired urinary tract infections in JNMC Hospital Aligarh, India. Ann Clin Microbiol Antimicrob 2007; 23: 6-14.

[13]. Bouskraoui M, Ait Sab I, Draiss G, Bourrous M, Sbihi M. Epidemiology of urinary tract infection in children in Marrakech. Arch Pediatr 2010; 17: 5177-8.6.

[14]. Al Sweih, N., W. Jamal and V.O. Rotimi. Spectrum and antibiotic resistance of uropathogens isolated from hospital and community patients with urinary tract infections in 2 large hospitals in Kuwait. 2005 Med. Princ. Practice, 14(6): 401-407. 
[15]. Gales AC, Jones RN, Turnidge J, RRennie, Ramphal R: Characterization of Pseudomonas aeruginosa isolates: occurrence rates, antimicrobial susceptibility patterns and molecular typing in the global sentry antimicrobial surveillance program 1997-1999. Clin Infect Dis 2001, 32(S1):146-155.

[16]. Vromen M, van der Van AJ, Knols AM, Stobberingh EE: Antimicrobial resistance patterns in urinary tract isolates from nursing homes residents. Fifteen years of data reviewed. J AntimicrobChemother1999, 44:113-116.

[17]. Kahlmeter G: Prevalence and antimicrobial susceptibility of pathogens in uncomplicated cystitis Europe. The ECO. SENS study. Int J Antimicrob Agents 2003, 22:49-52.

[18]. Dromigny JA, Nabeth P, Perrier Gros Claude JD: Distribution and susceptibility of bacterial urinary tract infections in Dakar, Senegal. Int J Antimicrob Agents 2002, 20:339-347.

[19]. Honderlick P, Cahen P, Gravisse J, Vignon D: Uncomplicated urinary tract infections, what about fosfomycin and nitrofurantoin in 2006? PatholBiol 2006, 54:462-6.

[20]. S. Manikandan, S. ganesapandian, Manoj Singh and A.K Kumaraguru. Emerging of Multidrug Resistance Pathogen from urinary tract infections. Current research in Bacteriology 2011, 